\title{
Mind- body therapies, telemedicine and psychophysical health in times of covid-19
}

\begin{abstract}
Introduction: Mind-body therapies encompass a series of practices that make use of meditation exercises, relaxation, mindfulness, controlled breathing, postures and smooth movements, in order to lead its practitioners to healthy psychophysical conditions. In times of quarantine and social distancing, online strategies are necessary to enable the access to healthcare. This work aims to present mind-body therapies, through telemedicine, as a resource for the decrease of number of injuries, and the promotion of health in times of quarantine and social distancing.
\end{abstract}

Methods: The ideas that are shown in this paper are based on contents of articles that had been published in scientific journals, and that had been complemented by the theoretical and practical knowledge of the authors in this area of knowledge.

Results: Studies show that online mind-body therapies can provide positive effects on physical, mental and emotional components that are related to health in different contexts.

Conclusion: In times of pandemic, mind-body therapies through telemedicine appear as important complementary resource of the strategies that aim the decrease of diseases and promotion of health, in situations of quarantine and social distancing.

Keywords: mental health, COVID-19, mind-body therapies, telemedicine, complementary and alternative medicine

\section{Introduction}

Since a significant proportion of the population started to experience situations of quarantine and social distancing that had been caused by the pandemic of coronavirus (COVID-19), it has become imperative that strategies for the decrease of diseases, and promotion of the physical and mental health of population, were fostered. ${ }^{1}$ In this scenery, Mind-Body Therapies (MBT) appear as a possibility of intervention that encompasses simplicity, diversity and effectiveness in the promotion of well-being, and its application shall be considered in the approaches of telemedicine.

MBT are a group of practices that are related to alternative, complementary and integrative (some of them that come from millennial, but others do not) that are secularized presented in the clinical context, such as: yoga, Qigong, meditation, hypnosis, biofeedback, progressive muscle relaxation, among others, whose effects have been broadly investigated in contexts of prevention of diseases, recovery and promotion of health. ${ }^{2}$ Nevertheless, most of the studies that had been held up, to the present moment, made use of the in-person approach.

The current situation of quarantine and social distancing that had been caused by the pandemic of Covid-19, showed to the world the need to establish new strategies of intervention, for the psychophysical health of the population, among them the approaches through telemedicine as a resource for the decrease of diseases and promotion of health in times of quarantine and social distancing.

\section{Discussion}

Quarantine and social distancing are measures that have been adopted by governmental authorities in order to, significantly reduce the circulation and agglomeration of people in public and private spaces, aiming to mitigate the spread of any infect contagious disease. However, changes in the individual day to day lives, and the social
Volume I4 Issue 3 - 202I

\author{
Paulo Rodrigo Santos Aristides, Ciro Oliveira \\ Queiroz, Cristiane Marques Pereira Xavier \\ Department of Physical Education, Bahiana School of Medicine \\ and Public Health, Brazil
}

Correspondence: Paulo Rodrigo Santos Aristides, Department of Physical Education, Bahiana School of Medicine and Public Health - Cabula, Brazil, Tel 71993976048, Email rodrigo.personalite@hotmail.com

Received: November 28, 2020 | Published: May 13, 2021 functioning, which are caused by such measures, are associated to a series of mental diseases that can involve mood disorders, anxiety, stress, depressive disorders, fear (contagion- disease-death), frustration, anger, boredom, insomnia, loneliness and worries about financial conditions, as well as food provision. These injuries to mental health can happen to people with past disorders and to individuals without any previous similar history, which can contribute for the increase in the global number of cases of suicide, domestic violence, self-mutilation, alcohol abuse and other risky behaviors, especially when the experience of quarantine and social distancing are prolonged..$^{3-14}$

Due to similar negative impact on psychophysical health caused by modern life, it has been some decades that the use of MBT by the world population has increased in social contexts, where the management of stress (and its unfolding) is necessary, not only in the clinical practice, aiming for the decrease of diseases, the recovery and promotion of patients' health. MBT use meditative exercises, controlled breathing, postures and smooth movements, aiming the relaxation that enables favorable mental, emotional and neurobiological conditions for the health recovery under a holistic perspective. ${ }^{2}$ The effectiveness of MBT has been investigated in many conditions of physical sickening (pain, fatigue, cancer, asthma, neurological and musculoskeletal matters), and negative symptoms of mental health (anxiety, depression, stress). The psychological and physical modifications promoted by MBT, have reverberated in better perceptions of health, well-being and quality of life in practitioners. ${ }^{15-23}$ In this sense, MBT appear as interesting therapeutical means, in times of quarantine and social distancing.

Aiming to reduce the negative impacts to health, under this context of the pandemic of Covid-19, many countries around the world are adopting strategies for the assistance to the population, and these strategies have significant influence on the perception of well-being and quality of life. Measures of economic support to the vulnerable 
ones, providing of instructions and material for hygiene and personal protection, emergency broadening of the number of hospital beds for critical patients with Covid-19, psychosocial consultations to patients and healthcare professionals, as well as to their families. ${ }^{1}$ However, taking into consideration the situation of the decrease of circulation and agglomeration of people, governmental efforts for the promotion of health shall look for innovative means to give assistance to the population that has been, directly or non-directly, affected. Then, the use of telemedicine to hold diagnoses, intervention and following up of the population in general, seems to be an excellent tool, once people facing social distancing, tend to actively look for online support to meet the demands (especially the ones on mental health), showing that this resource has good acceptance among the affected ones. ${ }^{24-28}$

Telemedicine (Telehealth or eHealth), is understood by a strategy where knowledge and provision of services in health are shared, by using telecommunication resources. This is not a new initiative, and it dates back to the 1960's. However, only in the 1990's, telemedicine was largely expanded, boosted by the technological development of medical devices and by the increase in the ability of telecommunication systems in transmitting information. This technical evolution, together with global transformations that redesigned the way the health services are accessed, ended up with the spread of projects in telemedicine, ${ }^{29-31}$ among which the use of online MBT as a type of intervention, has been gaining followers.

Recent studies highlighted the efficacy of the use of online MBT in the improvement of aspects of mental and physical health. In one of these studies, researchers observed that a group of patients with neurofibromatosis that had participated in an online MBT program, showed better results in the dimensions of Quality of Life (physical and psychological health social relations and environment) as well as better levels of anxiety, when compared to a placebo group. The intervention lasted 8 sessions, in which the intervention group with online MBT, had health assistance that involved 15 techniques of relaxation and whole attention (elicited), instructions for cognitive coping of stress and of medical symptoms, and teachings on personal growth and improvement. The placebo group received educational information on stress, symptoms of diseases, nutrition, exercise and communication with the medical team. The found outcomes remained for at least 6 months after the intervention. ${ }^{32}$

In another investigation, a group of researchers compared the effects of the intervention with Yoga and Tai chi, both on the online modality, on adults' physical health. Participants of each group did, on average, 135minutes per week of just one on the MBT (Yoga or Tai Chi) in the period of 12 weeks. By the end of the period of intervention, both groups showed improvement in the muscle strength and resistance, in the ability to hold tasks of functionality (dynamic balance and speed of walk), and in the flexibility, when compared to data from the baseline, with discreet advantage for the intervention group, with yoga in two parameters of strength (pushups and sit ups). ${ }^{33}$

Recently, researchers disclosed the strategies of care with MBT that had been proposed by a center of telemedicine, in the republic of Korea, which attends patients and survivors of Covid-19, their families and close people, as well as part of the population in general, who would have complaints on mental health that were related to the pandemic. This innovative measure had the writing of a manual with guidance so that doctors learned to stratify the profile of people that looked for the service of telemedicine, and to appoint the most adequate MBT for each case. Body-mind sessions were offered through videos on YouTube. The authors learned some lessons from this experience and presented important considerations to improve the strategy: 1) Use simple measures to evaluate mental health in the environment of telemedicine; 2) Respect the digital privacy of users; 3) Look for interactive platforms to provide the services of MBT, allowing more immediate dialogues as well as enabling online communitarian consultations ; 4) Respect the cultural characteristics of the communities, by offering MBT that do not disrespect social and personal beliefs; 5) Include in the scope of consultations, healthcare professionals that are in the frontline, during events such as the pandemic; 6)Develop more specific actions to each stressful situation, deciding between individual or collective approaches. ${ }^{34}$

These findings are particularly interesting when we observe that quarantine and social distancing can cause in the population, not only mental diseases, as well as a significant reduction in the levels of physical activity. It is known that physical and mental states are interdependent. Changed mental conditions cause neurobiological and behavioral changes, as much as body practices are, admittedly, able to influence the individual's perception of well-being, mediated by specific physiological and hormonal changes. ${ }^{35-40}$

We need to take into consideration that in the current emergency context, the junction between telemedicine and MBT can be developed under the perspective of research-action, to the extent that at the same time it offers care to their services' users, it also broadens the knowledge on cost-effectiveness by this type of intervention. Despite a large number of scientific papers have shown beneficial effects of MBT that are offered through telemedicine, it is not clear yet how and which types of MBT can be selected in programs for the decrease of diseases, and the promotion of health in different psychophysical conditions, cultures and contexts. It is also necessary to improve the understanding on the relation dosage-answer of the practices of MBT, in which concerns frequency, duration and intensity. There are still few works that show the continuum between: adherence to the online programs of MBT, the perception of self- efficacy during the interventions and the adherence to these programs on a virtual environment.

\section{Limitations}

Among the limitations in this work, we highlight the fact that the scientific literature does not approach the possible adverse effects related to the difficulties in holding interventions with MBT on a virtual environment, such as: feeling of irritation or hopelessness caused by the interruption in the internet signal during the sessions, and possible physical injuries caused by the impossibility of kinesthetic guidelines.

\section{Conclusion}

Many authors believe that there will be new pandemics related to physical inactivity and injuries to mental health because of the pandemic of Covid-19, which can overload health systems, negatively impact the social relations, cause the increase of absenteeism, among other consequences. Then, considering the information presented in this study, we suggest that the governmental authorities adopt, in their health systems, MBT services through telemedicine as a complementary resource to the strategies that aim to mitigate the disastrous consequences on the psychophysical health that are caused by the quarantine and social distancing.

\section{Acknowledgments}

None.

\section{Conflicts of interest}

None. Author has no pertinent financial interests with the subject matter or materials discussed. 


\section{Funding}

None.

\section{References}

1. Usher K, Bhullar N, Jackson D. Life in the pandemic: Social isolation and mental health. J Clin Nurs. 2020;29(15-16):2756-2757.

2. Dossett ML, Fricchione GL, Benson H. A new era for mind-body medicine. N Engl J Med. 2020;382(15):1390.

3. Bai YM, Lin CC, Lin CY, et al. Survey of stress reactions among health care workers involved with the SARS outbreak. Psychiatr Serv. 2004;55(9):1055-1057.

4. Brooks SK, Webster RK, Smith LE, et al. The psychological impact of quarantine and how to reduce it: rapid review of the evidence. Lancet. 2020;395(10227):912-920.

5. Cava MA, Fay KE, Beanlands HJ, et al. The experience of quarantine for individuals affected by SARS in Toronto. Public Health Nurs. 2005;22(5):398-406

6. Desclaux A, Badji D, Ndione AG, et al. Accepted monitoring or endured quarantine? Ebola contacts' perceptions in Senegal. Soc Sci Me. 2017;178:38-45.

7. Hawryluck L, Gold WL, Robinson S, et al. SARS control and psychological effects of quarantine, Toronto, Canada. Emerg Infect Dis. 2004;10(7):1206-1212.

8. Taylor R, Taylor H, Nguyen A, et al. Social isolation from family and friends and mental health among African Americans and Black Caribbeans. Am J Orthopsychiatry. 2020;90(4):468-478.

9. Taylor $\mathrm{OH}$, Taylor JR, Nguyen WA. Social Isolation, Depression, and Psychological Distress among Older Adults. Physiol Behav. 2018;176(1):139-q48.

10. Moukaddam N, Shah A. Psychiatrists Beware! The Impact of COVID-19 and Pandemics on Mental Health. Psychiatric Times. 2020;37(3)

11. Li W, Yang Y, Liu ZH, et al. Progression of Mental Health Services during the COVID-19 Outbreak in China. Int J Biol Sci. 2020;16(10):1732-1738.

12. Monica S, Corporation R. Public Mental Health Crisis during COVID19 Pandemic, China. Emerg Infect Dis. 2020;26(7):1616-1618.

13. Jakovljevic M, Bjedov S, Jaksic N, et al. Covid-19 Pandemia and Public and Global Mental Health From the Perspective of Global Health Security. Psychiatr Danub. 2020;32(1):6-14.

14. Choi KR, Heilemann M V, Fauer A, et al. A Second Pandemic: Menta Health Spillover From the Novel Coronavirus (COVID-19). J Am Psychiatr Nurses Assoc. 2020;1078390320919803.

15. Love MF et al. Mind-Body Interventions, Psychological Stressors, and Quality of Life in Stroke Survivors. Stroke. 2019;50(2):434-440.

16. Laird KT el al. Mind-Body Therapies for Late-Life Mental and Cognitive Health. Curr Psychiatry Rep. 2018;20(1):2.

17. Wang X. The Effect of Mind-Body Therapies on Insomnia: A Systematic Review and Meta-Analysis. Evid Based Complement Alternat Med. 2019;2019:9359807.

18. Carlson LE, Zelinski E, Toivonen K, et al. Mind-Body Therapies in Cancer: What Is the Latest Evidence? Curr Oncol Rep. 2017;19(10):67.

19. Asher GN, Gerkin J, Gaynes BN. Complementary Therapies for Mental Health Disorders. Med Clin North Am. 2017;101(5):847-864.

20. Ratarasarn K, Kundu A. Yoga and Tai Chi: a mind-body approach in managing respiratory symptoms in obstructive lung diseases. Curr Opin Pulm Med. 2020;26(2):186-192.

21. Wahbeh H, Elsas SM, Oken BS. Mind-body interventions: Applications in neurology. Neurology. 2008;70(24):2321-2318
22. Jacquart J, Miller K, Radossi A, et al. The effectiveness of a communitybased, mind-body group for symptoms of depression and anxiety. $A d v$ Mind Body Med. 2014;28(3):6-13.

23. Burnett-Zeigler I, Schuette S, Victorson D, et al. Mind-body approaches to treating mental health symptoms among disadvantaged populations: A comprehensive review. J Altern Complement Med. 2016;22(2):115-124.

24. Liu S, Yang L, Zhang C, et al. Online mental health services in China during the COVID-19 outbreak. The Lancet Psychiatry. 2020;7(4):e17e18.

25. Zhou X, Snoswell CL, Harding LE, et al. The Role of Telehealth in Reducing the Mental Health Burden from COVID-19. Telemed $J E$ Health. 2020;26(4):377-379.

26. Ohannessian R, Duong TA, Anna Odone A. Global Telemedicine Implementation and Integration Within Health Systems to Fight the COVID-19 Pandemic: A Call to Action. JMIR Public Health Surveill. 2020;6(2):e18810

27. Fisk M, Livingstone A, Pit SW. Telehealth in the Context of COVID-19: Changing Perspectives in Australia, the United Kingdom, and the United States. J Med Internet Res. 2020;22(6):e19264.

28. Caetano R, Silva BA, Menezes Guedes CCA, et al. Challenges and opportunities for telehealth during the COVID-19 pandemic: ideas on spaces and initiatives in the Brazilian context. Cad Saúde Pública. 2020;36(5):e00088920.

29. Zajtchuk R, Gilbert GR. Telemedicine : A New Dimension the Practice of Medicine. Dis Mon. 1999;45(6):197-262.

30. Thrall JH, Boland G. Telemedicine in practice. Semin Nucl Med. 1998;28(2):145-157.

31. https://www.who.int/ehealth/about/en/

32. Vranceanu AM, Riklin E, Merker VL, et al. Mind-body therapy via videoconferencing in patients with neurofibromatosis. Neurology. 2016;87(8):806-814.

33. Martin AC, Candow D. Effects of Online Yoga and Tai Chi on Physical Health Outcome Measures of Adult Informal Caregivers. Int $J$ Yoga. 2019;12(1):37-44.

34. Chan-Young K, Hui-Yong K, Jong W K. Using Mind-Body Modalities via Telemedicine during the COVID-19 Crisis: Cases in the Republic of Korea. Int J Environ Res Public Health. 2020;17(12):4477.

35. Ammar A, Brach M, Trabelsi K, et al. Effects of COVID-19 Home Confinement on Eating Behaviour and Physical Activity: Results of the ECLB-COVID19 International Online Survey. Nutrients. 2020;12(6):1583.

36. Ruiz-Roso MB, Torcal CK, Escalante DCM, et al. COVID-19 Lockdown and Changes of the Dietary Pattern and Physical Activity Habits in a Cohort of Patients with Type 2 Diabetes Mellitus. Nutrients. 2020;12(8):2327.

37. Lesser IA, Nienhuis CP. The Impact of COVID-19 on Physical Activity Behavior and Well-Being of Canadians. Int J Environ Res Public Health. 2020;17(11):3899.

38. Stanton R, To QG, Khalesi S, et al. Depression, Anxiety and Stress during COVID-19: Associations with Changes in Physical Activity, Sleep Tobacco and Alcohol Use in Australian Adults. Int J Environ Res Public Health. 2020;17(11):4065.

39. Goethals L, et al. Impact of Home Quarantine on Physical Activity Among Older Adults Living at Home During the COVID-19 Pandemic: Qualitative Interview Study. JMIR Aging. 2020;3(1):e19007.

40. Duncan GE, et al. Perceived change in physical activity levels and mental health during COVID-19: Findings among adult twin pairs. PLoS One. 2020;15(8):e0237695. 